\title{
CoTrRank: Trust Evaluation of Users and Tweets
}

\author{
Peiyao $\mathrm{Li}^{1}$, Weiliang Zhao ${ }^{1}$, Jian Yang ${ }^{1,2 *}$ and $\mathbf{J i a} \mathbf{W u}^{1}$ \\ ${ }^{1}$ Department of Computing, Macquarie University, NSW 2113, Australia \\ ${ }^{2}$ School of Computer Science, DongHua University, ShangHai, China \\ peiyao.li@students.mq.edu.au, \{weiliang.zhao, jian.yang, jia.wu\}@mq.edu.au
}

\begin{abstract}
Trust evaluation of people and information on Twitter is critical for maintaining a healthy online social environment. How to evaluate the trustworthiness of users and tweets becomes a challenging question. In this demo, we show how our proposed CoTrRank approach deal with this problem. This approach models users and tweets in two coupled networks and calculate their trust values in different trust spaces. In particular, our solution provides a configurable way when mapping the calculated raw evidences to the trust values. The CoTrRank demo system has an interactive interface to show how our proposed approach produces more effective and adaptive trust evaluation results comparing with baseline methods.
\end{abstract}

\section{Introduction}

Twitter nowadays is a popular social media platform for information dissemination by people, business, and government agencies. It is important to have mechanisms in place to evaluate the trustworthiness of users and their posts to provide a healthy online social environment. We assign trust values to users and tweets to indicate their trustworthy degrees based on a generic trust principle: A user who posts or mentions by more trustworthy tweets and is followed by more trustworthy users is more trustworthy; a tweet that is posted by a more trustworthy user and retweeted/replied by more trustworthy tweets is more trustworthy. To effectively calculate the trust values of users and tweets, we need to consider: (1) how to model various user/tweet relations/interactions; (2) how to calculate the trust values of users and tweets that are in different trust spaces but linked with each other; (3) how to determine users/tweets trustworthy degrees under different situations.

Existing approaches attempt to evaluate trust by analysing Twitter as a heterogeneous network with users and tweets as nodes and their relations as links [Weng et al., 2010; Yamaguchi et al., 2010; Hsu et al., 2017]. However, most of these methods are based on network models constructed with

\footnotetext{
${ }^{*}$ Contact Author
}

limited types of interactions, such as follow and retweet, using a single network consisting of both user nodes and tweet nodes [Yamaguchi et al., 2010]. These network models have their limitations in capturing the complex and complicated relations among/between users and tweets [Li et al., 2018]. In this work, we develop a configurable trust ranking approach (CoTrRank) based on a coupled dual networks model. We put users and tweets in two networks and scrutinise the coupling effect between them. The trust values are calculated with the consideration of their real meanings in different trust spaces. The experimental results show that the proposed CoTrRank provides more accurate evaluations of the trustworthiness of users and tweets comparing with baseline methods as PageRank [Page et al., 1999], TURank [Yamaguchi et al., 2010], and CoRank [Li et al., 2018].

\section{CoTrRank Trust Evaluation}

In Twitter, there is a variety of interactions between/among users and tweets. We construct a user network and a tweet network. The user network is built up with users as nodes and follow relations as edges; the tweet network is built up with tweets as nodes and retweet, reply relations as edges. These two networks are coupled with each other by links representing the mention and bipartite post relations. The trust values of users and tweets are related with their linked users and tweets through different relations.

The process of the proposed CoTrRank approach is shown in Fig. 1. Matrix $\mathbf{M}$ is the the weight matrix for calculating trust values of users according to follow relations. Matrix $\mathbf{N}$ is the weight matrix for calculating trust values of tweets according to retweet and reply relations. Matrix $\mathbf{W}$ is the weight matrix for calculating trust values of users according to tweets that mention them or posted by them. Matrix V is the weight matrix for tweets according to users who post them. The trust values of users and that of tweets are stored in two vectors $\boldsymbol{X}$ and $\boldsymbol{Y}$, respectively. The proposed method considers the coupling effect between the user network and the tweet network as follows:

- A user $u_{i}$ 's trust value $x_{i}$ is calculated according to the trust values of $u_{i}$ 's followers, the trust values of tweets that are posted by $u_{i}$, and the trust values of tweets that mention $u_{i}, x_{i} \in \boldsymbol{X}$;

- A tweet $t_{i}$ 's trust value $y_{i}$ is calculated according to the 


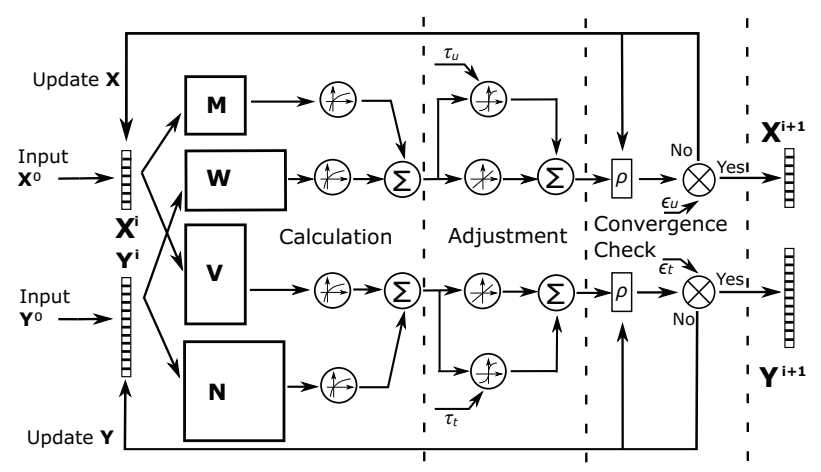

Figure 1: Process of CoTrRank trust evaluation.

trust values of tweets that retweet or reply to $t_{i}$ and the trust value of the user who posts $t_{i}, y_{i} \in \boldsymbol{Y}$.

In such a way, trust values of users and tweets are dependent on each other based on the interactions that couple the user network and the tweet network. We propose the following formulas to calculate the trust values of each user and tweet:

$$
\begin{aligned}
x_{i} & =(\alpha) \mathcal{F}\left(\sum_{j} m_{i, j} x_{j}\right)+(1-\alpha) \mathcal{F}\left(\sum_{k} w_{i, k} y_{k}\right) \\
y_{i} & =(\beta) \mathcal{F}\left(\sum_{j} n_{i, j} y_{j}\right)+(1-\beta) \mathcal{F}\left(v_{i, k} x_{k}\right)
\end{aligned}
$$

where $\alpha$ and $\beta$ are control parameters to specify the proportion of contributions from nodes in the user network and the tweet network. Here, $m_{i, j} \in \mathbf{M}, w_{i, k} \in \mathbf{W}, n_{i, j} \in \mathbf{N}$, and $v_{i, k} \in \mathbf{V}$. Function $\mathcal{F}()$ is a logarithmic function that scales the summation of the weighted values of one's related nodes in each network.

As one choice, the user trust values $\boldsymbol{X}$ and tweet trust values $\boldsymbol{Y}$ calculated by Eq. (1) and (2) are normalised by dividing their corresponding sums. However, this widely used normalisation over sum method [Yan et al., 2012] makes users and tweets with high trust values dominate the trust evaluation. As another choice, we employ Sigmoid functions [Li et al., 2018] to perform trust value mapping that benefits users and tweets linked with a large number of nodes with medium trust degrees. To deal with different trust evaluation situations, we propose a configurable trust mapping method using a weighted integration of the normalised values and the Sigmoid mapped values of users and tweets. We can make users/tweets with high trust degrees have more impact on the trust evaluation results or users/tweets with medium trust degrees have more impact on the trust evaluation results.

\section{Demo}

We built a demonstration system with an interactive interface. Figure 2 shows the screenshots of our demonstration system ${ }^{1}$. This system allows user to input the control parameters used in Eq. (1) and (2), and specify the weights in the mapping configuration. Users can input one user id or multiple user ids in the 'UserID' field, and the demo system can provide trust values calculated under the specific settings.

\footnotetext{
${ }^{1}$ A demo video can be viewed at: https://youtu.be/eeiMVO4qS1s
}
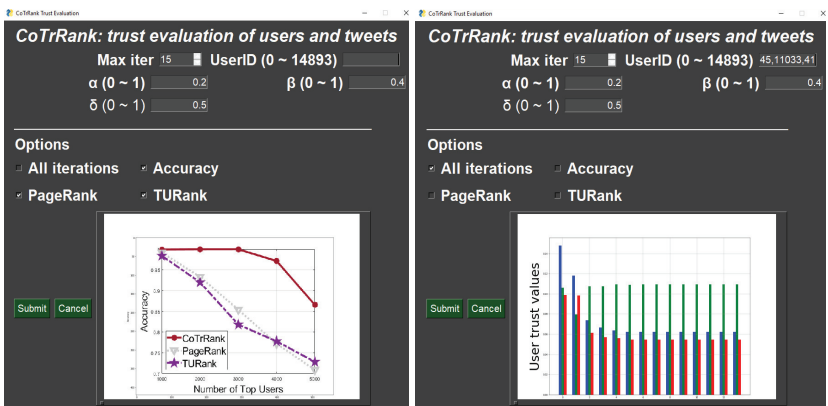

Figure 2: Screenshots of our demonstration system.

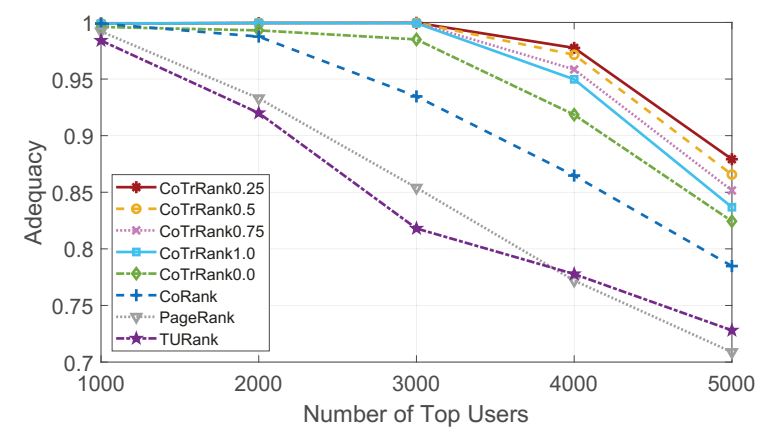

Figure 3: Adequacy comparison between CoTrRank with different mapping configurations and baseline methods.

If the 'all iterations' box is checked, the system will display the trust values in each round of iteration; otherwise, the system will only provide the final trust values. The right window in Fig. 2 shows the trust values of three users calculated by CoTrRank in all iterations. If the 'adequacy' checkbox is checked, the demo system will display the adequacy results of the proposed CoTrRank with the parameter settings specified in the parameter section. User can also check the 'PageRank' and/or 'TURank' checkbox to show the adequacy results of these baseline methods. The left window in Fig. 2 shows an example of the adequacy outputs of our proposed CoTrRank method and two baseline methods.

In our demonstration system, we use the dataset collected from Twitter based on the Tasmania community ${ }^{2}$. In this dataset, there are approximately 10,000 users and one million tweets that have relations between/among other users and/or tweets in this dataset. We invite human examiners to select 4,766 users that are considered to be more trustworthy comparing with other users in the dataset. The evaluation metrics in [Heidemann et al., 2010] are utilised to evaluate the adequacy of our proposed approach and the baseline methods. The results are shown in Fig. 3. The proposed CoTrRank approach with Sigmoid mapping has outperformed that with normalisation over sum. By combining the Sigmoid mapping and the normalisation over sum method, the CoTrRank approach has achieved better adequacy results. Our CoTrRank methods with different configurations in trust value mapping have outperformed the three baselines methods.

\footnotetext{
${ }^{2}$ The dataset and source codes are available at: https://github.com/TrustEval/Twitter_Tas_dataset
} 


\section{References}

[Heidemann et al., 2010] Julia Heidemann, Mathias Klier, and Florian Probst. Identifying key users in online social networks: A pagerank based approach. In 31st International Conference on Information Systems (ICIS), 2010.

[Hsu et al., 2017] Chin-Chi Hsu, Yi-An Lai, Wen-Hao Chen, Ming-Han Feng, and Shou-De Lin. Unsupervised ranking using graph structures and node attributes. In Proceedings of the Tenth ACM International Conference on Web Search and Data Mining, WSDM '17, pages 771-779, 2017.

[Li et al., 2018] Peiyao Li, Weiliang Zhao, and Jian Yang. Corank: A coupled dual networks approach to trust evaluation on twitter (forthcoming). In Proceedings of the 19th International Conference on Web Information Systems Engineering (WISE'18), 2018.

[Page et al., 1999] Lawrence Page, Sergey Brin, Rajeev Motwani, and Terry Winograd. The pagerank citation ranking: Bringing order to the web. Technical Report 1999-66, November 1999.

[Weng et al., 2010] Jianshu Weng, Ee-Peng Lim, Jing Jiang, and Qi He. Twitterrank: Finding topic-sensitive influential twitterers. In Proceedings of the Third ACM International Conference on Web Search and Data Mining, WSDM '10, pages 261-270, 2010.

[Yamaguchi et al., 2010] Yuto Yamaguchi, Tsubasa Takahashi, Toshiyuki Amagasa, and Hiroyuki Kitagawa. TURank: Twitter user ranking based on user-tweet graph analysis. Lecture Notes in Computer Science, 6488 LNCS:240-253, 2010.

[Yan et al., 2012] Rui Yan, Mirella Lapata, and Xiaoming Li. Tweet recommendation with graph co-ranking. In Proceedings of the 50th Annual Meeting of the Association for Computational Linguistics: Long Papers - Volume 1, ACL '12, pages 516-525, 2012. 